sivität auch über die Fachgrenzen hinweg eine für die weitere Arbeit am Thema der theologischen Anthropologie wichtige Bestandsaufnahme. Es zeigt darüber hinaus, dass eine Festlegung des Menschseins gerade da durchbrochen wird, wo die Verheißungen Gottes an Israel und die Kirche erinnert werden (261-277).

Der Reichtum biblischen Sprechens vom Menschen, wie er etwa von $B$. Janowski beispielhaft vorgeführt wird, kann dabei die Beschränkungen und Aporien aufbrechen, die mit den gängigen anthropologischen Begriffen verbunden sind. Dabei wird nicht eine allgemeine Theorie vom Menschen anvisiert, sondern in der Konzentration auf Lob und Klage werden die Fragen nach dem Verstehen des menschlichen Lebens vor Gott bearbeitet. Was in der genauen Betrachtung der einzelnen Psalmen entdeckt und beschrieben werden kann, eröffnet die Möglichkeit, diesen Wahrnehmungen des Lebens nachzugehen, die schon von ihren grundlegenden Kategorien her oft quer zu den eingespielten anthropologischen Konzepten liegen. Gerade der Verzicht auf eine Vereinheitlichung der Vielfalt biblischen Sprechens vom Menschen bringt ihre Erfahrungsnähe zur Geltung. Das Aufsuchen der biblischen Perspektiven gehört darum zu den wichtigsten und lohnendsten Aufgaben der theologischen Anthropologie.

\title{
Eucharistie oder Herrenmahl? Liturgiewissenschaft und Kirchengeschichte im Gespräch Martin Wallraff
}

Ulrich Kühn, Der eucharistische Charakter des Herrenmahls: PTh 88 (1999) 255-268. - Michael Meyer-Blanck, Liturgiewissenschaft und Kirche. Eine ökumenische Verhältnisbestimmung in zehn Thesen: Michael Meyer-Blanck (Hg.), Liturgiewissenschaft und Kirche. Ökumenische Perspektiven, cmz Rheinbach 2003, 111-138. - Klaus Raschzok, Der Streit um das Eucharistiegebet in den Kirchen der Reformation: Winfried Haunerland (Hg.), Mehr als Brot und Wein. Theologische Kontexte der Eucharistie, Echter Würzburg 2005, 145-172. - HansChristoph Schmidt-Lauber/Frieder Schulz, Kerygmatisches oder eucharistisches Abendmahlsverständnis? Antwort auf eine kritische Herausforderung der gegenwärtigen Liturgiewissenschaft: LJ 49 (1999) 93-114. - Frieder Schulz, Eingrenzung oder Ausstrahlung?: Michael Meyer-Blanck (Hg.), Liturgiewissenschaft und Kirche, Ökumenische Perspektiven, cmz Rheinbach 2003, 91-107. - Dorothea Wendebourg, Den falschen Weg Roms zu Ende gegangen? Zur gegenwärtigen Diskussion über Martin Luthers Gottesdienstreform und ihr Verhältnis zu den Traditionen der Alten Kirche: ZThK 94 (1997) 437-467. - Dorothea Wendebourg, Noch einmal „Den falschen Weg Roms zu Ende gegangen?“ Auseinandersetzung mit meinen Kritikern: ZThK 99 (2002) 400-440 (zit. als II).

In den vergangenen Jahren ist es nicht sehr häufig geschehen, dass theologische Themen wirklich in direktem Austausch der Argumente zwischen kontrastierenden Positionen verhandelt wurden, also diskutiert im eigentlichen Sinne des Wortes. Statt dessen ergibt sich in vielen Fällen das Bild der Forschungs-„,dis- 


\section{Eucharistie oder Herrenmahl? Liturgiewissenschaft und Kirchengeschichte im Gespräch \\ Martin Wallraff}

Ulrich Kühn, Der eucharistische Charakter des Herrenmahls: PTh 88 (1999) 255-268. - Michael Meyer-Blanck, Liturgiewissenschaft und Kirche. Eine ökumenische Verhältnisbestimmung in zehn Thesen: Michael Meyer-Blanck (Hg.), Liturgiewissenschaft und Kirche. Ökumenische Perspektiven, cmz Rheinbach 2003, 111-138. - Klaus Raschzok, Der Streit um das Eucharistiegebet in den Kirchen der Reformation: Winfried Haunerland (Hg.), Mehr als Brot und Wein. Theologische Kontexte der Eucharistie, Echter Würzburg 2005, 145-172. - HansChristoph Schmidt-Lauber/Frieder Schulz, Kerygmatisches oder eucharistisches Abendmahlsverständnis? Antwort auf eine kritische Herausforderung der gegenwärtigen Liturgiewissenschaft: LJ 49 (1999) 93-114. - Frieder Schulz, Eingrenzung oder Ausstrahlung?: Michael Meyer-Blanck (Hg.), Liturgiewissenschaft und Kirche, Ökumenische Perspektiven, cmz Rheinbach 2003, 91-107. - Dorothea Wendebourg, Den falschen Weg Roms zu Ende gegangen? Zur gegenwärtigen Diskussion über Martin Luthers Gottesdienstreform und ihr Verhältnis zu den Traditionen der Alten Kirche: ZThK 94 (1997) 437-467. - Dorothea Wendebourg, Noch einmal „Den falschen Weg Roms zu Ende gegangen?“ Auseinandersetzung mit meinen Kritikern: ZThK 99 (2002) 400-440 (zit. als II).

In den vergangenen Jahren ist es nicht sehr häufig geschehen, dass theologische Themen wirklich in direktem Austausch der Argumente zwischen kontrastierenden Positionen verhandelt wurden, also diskutiert im eigentlichen Sinne des Wortes. Statt dessen ergibt sich in vielen Fällen das Bild der Forschungs-„dis-

Verkündigung und Forschung 51. Jg., Heft 2, S. 55-63

ISSN 0342-2410 CC Chr. Kaiser / Gütersloher Verlagshaus, 2006

Brought to you by | Universitaetsbibliothek Basel 
kussion" eher aus der Addition etwas unterschiedlich nuancierter Farbtupfer, die in mehr oder minder geringem zeitlichen Abstand aneinander gesetzt werden und so im Laufe der Zeit ein je neues Panorama ergeben. Auslöser einer viel beachteten Diskussion im besagten engen Sinne war die Tübinger Antrittsvorlesung der Kirchenhistorikerin D. Wendebourg aus dem Jahr 1997. Es ging darin um Theologie und Liturgie des Abendmahls, und der Debatte war nicht zuletzt deshalb ein hohes Maß an Aufmerksamkeit (und konkreter Wirkung) sicher, weil sie in die Abschlussphase der Arbeiten am neuen „Evangelischen Gottesdienstbuch" (Berlin 1999) fiel.

Der Beitrag ging aus von der Feststellung, „daß - zumal im deutschen evangelischen Raum - die Arbeit der Kirchenhistoriker und die der Fachleute für liturgische Fragen [...] weitgehend nebeneinander herlaufen" (441). Angesichts dieser Sachlage verstand sich der Aufsatz als Beitrag zum Gespräch zwischen den Disziplinen Kirchengeschichte und Liturgiewissenschaft. In der Diskussion ist er als solcher jedoch kaum zur Geltung gekommen. Die Reaktionen kamen aus dem Bereich der Systematischen Theologie (Kühn) sowie vor allem aus der Praktischen Theologie (Schmidt-Lauber, Schulz, Meyer-Blanck, Raschzok), wobei jedoch nicht in allen Fällen die Debatte als Einladung zu einem vertieften Gespräch mit der historischen Theologie genutzt wurde. Dagegen hat sich meiner Kenntnis nach kein einziger „zünftiger“ Kirchenhistoriker zu Wort gemeldet, obgleich die Thesen von Wendebourg für dessen Disziplin eine Fülle von Anregungen bieten.

$\mathrm{Zu}$ den weiteren Eigentümlichkeiten der Diskussion gehörte es, dass in kurzer Zeit diverse Stellungnahmen publiziert wurden, die alle eines gemeinsam hatten: die mehr oder minder differenzierte Ablehnung der wendebourgschen Thesen (indirekte Unterstützung erhält Wendebourgs Position z. B. durch R. Schwarz und N. Slenczka, s. S. 29 f.). Es mag in der Natur der Sache liegen, dass Widerspruch stärker zur öffentlichen Reaktion reizt als Zustimmung, doch hätte man sich ohne Weiteres vorstellen können, dass solcher Widerspruch seinerseits Reaktionen hervorrufen und damit ein buntes Bild entstehen lassen könnte. Es gab indessen, soweit ich sehen kann, nur einen einzigen Beitrag zur Verteidigung der ursprünglichen Thesen, und dieser Beitrag stammte wiederum von der ursprünglichen Verfasserin. Es kann dennoch kein Zweifel sein, dass es um mehr geht als um eine theologische Partikularposition und dass die vorgebrachten Argumente (die zum Teil in ihrer Differenziertheit kaum wahrgenommen wurden) mehr sind als ad-hoc-Konstruktionen zu deren Verteidigung.

Im Folgenden kann nicht mehr geboten werden als eine knappe Zusammenfassung nur der wichtigsten Stimmen (detailliertere Informationen bei Raschzok) - gefolgt von einigen Schlussbemerkungen, die den Ertrag bündeln. Auch wenn dies aus spezifisch kirchenhistorischer Sicht geschieht, reicht der Raum nicht, um eine eigenständige Stellungnahme aus dieser Perspektive argumentativ zu entfalten.

Zunächst also: „Den falschen Weg Roms zu Ende gegangen?“ - der vor fast 
10 Jahren erschienene Aufsatz von D. Wendebourg. Der Titel möchte in Kurzform die communis opinio über Rolle und Stellung der liturgischen Veränderungen beim Abendmahl durch die Wittenberger Reformation referieren, und das Fragezeichen drückt aus, dass Wendebourg diese (wesentlich von H.-Ch. Schmidt-Lauber und F. Schulz geprägte) communis opinio in Frage stellen möchte. Dieser bestrittenen Meinung zufolge sei Martin Luther bei seinen Reformvorschlägen viel stärker der Tradition „Roms“, also des lateinischen Westens seit dem Ausgang der Antike, verpflichtet gewesen, als ihm selbst bewusst war und als die explizite Abgrenzung gegen die mittelalterliche Messe vermuten ließe. Das gelte vor allem für die Konzentration auf, ja die völlige Isolation der Einsetzungsworte als Konstituens des Abendmahls. Dass er somit ganz in der Logik des lateinischen Erbes gedacht und gehandelt habe, ja, dass er dieses Erbe radikalisiert und „zu Ende gebracht“ habe, sei vor allem deshalb fatal, weil der Weg des lateinischen Westens schon seit langem ein Irrweg gewesen sei. Wenn man so denkt, dann folgt tatsächlich mehr daraus als „nur eine historische Theorie“; damit ist „vielmehr zugleich ein ökumenisches Programm“ gegeben (441), denn man muss ja dann versuchen, die Fehlentwicklung rückgängig zu machen, und zwar geschieht dies in aller Regel unter Anknüpfung an ältere historische Strata, solche, die den Kirchentrennungen voraus liegen und daher als ökumenisch besonders verträglich gelten. Dieses Programm charakterisiert Wendebourg mit dem (nicht allzu schönen, ja bewusst polemischen) Neologismus „Eucharistisierung “ - unter Aufnahme des in den ersten drei Jahrhunderten vorherrschenden Terminus eucharistia (Danksagung, zuerst Did 9,5) für das Abendmahl.

Gegen diese communis opinio und damit gegen das „ökumenische Programm“ richtet sich doppelter Widerspruch: Zum einen geht es darum, ob der „Weg Roms“ wirklich als gänzlich falsch zu bewerten ist oder nicht vielmehr in gewissen Aspekten als sachgemäße Entfaltung des im Abendmahl Intendierten; zum anderen wird gefragt, ob nicht Luthers Reformwerk in höherem Maße als selbstständig und daher kritisch und innovativ dem Vorausgehenden gegenüber $\mathrm{zu}$ sehen ist. In diese beiden Hauptteile - einen altkirchlichen und einen reformationshistorischen - ist der Aufsatz gegliedert. Für beide Aspekte spielen die Einsetzungsworte - ihre liturgische Position und theologische Funktion - eine herausgehobene Rolle; auf deren Interpretation richtet sich das Hauptaugenmerk.

Der erste Teil beginnt mit der Feststellung: „Daß die Einsetzungsworte zur zentralen religiösen Mahlfeier der Kirche gehören, ist keineswegs überall, vermutlich aber nirgends von Anfang an der Fall“ (441) - eine Sichtweise übrigens, die unter Spezialisten längst nicht mehr auf Verwunderung oder gar Widerspruch stößt, die aber im vorliegenden Zusammenhang dennoch als „neu“ und „spannend“ wahrgenommen wurde (II, 420). Vielmehr sind die Einsetzungsworte zuerst in der „Traditio apostolica“ ca. zwei Jahrhunderte nach den neutestamentlichen Schriften in der Mahlfeier belegt (442, nebenbei: Weder, dass der Text „aus dem frühen 3. Jahrhundert“ stammt, noch dass er „meist Hippolyt von 
Rom zugeschrieben wird“, entspricht dem heutigen Forschungskonsens). Eine steile liturgische Karriere machte der Bericht von der Einsetzung des Mahles aber erst im 4. Jh., wir finden ihn jetzt in beinahe allen überlieferten Riten - auch dies so weit unbestritten. Die Frage ist nun: Warum wurden diese Worte in die Mahlfeier integriert? Und welche Bedeutung erhielten sie dort?

Auf die erste Frage antwortet Wendebourg, dass der Einsetzungsbericht das explizit macht, was implizit der Mahlfeier schon immer zugrunde liegt: „die Feier bringt nun selbst zum Ausdruck, warum sie vollzogen wird, wie sie legitimiert ist" (445). Tatsächlich findet man bei Justin (1 apol. 65f.) die Situation, dass die Einsetzung als „Begründung und Erklärung“ (445) eine Rolle spielt, aber wie es scheint - im Modus der katechetischen Unterweisung, nicht im Modus der liturgischen Rezitation. Als sie in die Feier selbst eindrangen, wurde diesen Worten sogleich „konsekratorische Kraft und Funktion zugeschrieben“ (448). Das ist in massiver Form bei Ambrosius von Mailand tatsächlich greifbar, spätere lateinische Autoren folgten ihm; insofern mag es wohl sein, dass der „Weg Roms“ in Wirklichkeit in Mailand beginnt. Für den Osten beruft sich Wendebourg auf eine öfters zitierte Chrysostomos-Stelle (De proditione Judae hom. $1,6=$ PG 49, 380). Man wird fragen können, ob diese gelegentliche Predigtäußerung die große Last der theologischen Argumentation allein tragen kann; weitere Belege aus dem 4. Jh. finden sich jedenfalls nicht. Dankenswerterweise wird der Befund in einer sehr langen Anmerkung (Anm. 41 über drei Seiten!, s. aber auch Anm. 52) entfaltet; dabei wird deutlich, wie komplex die Sachlage ist und wie sehr sie nach einer eigenen, differenzierteren Untersuchung verlangte.

Wo jedenfalls - und darauf kommt es an - die Einsetzungsworte eingeführt wurden und wo ihnen konsekratorische Kraft zugeschrieben wurde, da bringen sie zum Ausdruck, dass die Mahlfeier nicht mehr primär von der feiernden Gemeinde Gott dargebrachte Dankeshandlung ist, sondern wesentlich Handeln Gottes, der sich selbst schenkt und für die Gemeinde gegenwärtig wird (451). Insofern ist die Einfügung der Einsetzungsworte nicht nur legitim und sachgemäß, sondern geradezu zwingend: Es handelt sich um einen „Prozeß der Selbstklärung, in dem liturgisch und theologisch herausgearbeitet wurde, was die christliche Abendmahlsfeier von jeher bestimmte“ (452). Freilich blieben diese Worte immer ein Fremdkörper in dem Gebetskontext, in dem sie nun zu stehen kommen: In dem lobpreisenden Gebet, in dem die versammelte Gemeinde sich an Gott wendet, findet ein jäher Sprecherwechsel statt, der die Kommunikationssituation radikal verändert: „Das ist mein Leib“ - nun spricht Christus zu seiner Gemeinde, Christus, der jetzt gegenwärtig wird und sich selbst schenkt: "Nehmt und eßt alle davon“ (447).

Gerade an diesem Bruch setzt für Wendebourg die Deutung der Liturgiereform Luthers an. Die Einsetzungsworte streifen den sie umgebenden Gebetszusammenhang wie das Küken die Eierschalen ab; der grammatische und theologische Bruch wird beseitigt, und „so kommt der Bericht ungebrochen als Bericht zu Gehör“ (459f.). Luther steht nun tatsächlich in Kontinuität zum bis- 
herigen Weg der lateinischen Tradition (die freilich nicht durchweg als Irrweg erscheint), insofern er an die immer weiter fortschreitende Konzentration auf die Einsetzungsworte als entscheidendes Moment (des „operative“ effect“, wie Wendebourg mit G. Dix sagt [451], der „Wandlung“, wie die mittelalterliche Tradition sagt) anknüpft, ja diese Worte isoliert und als im Grunde alleiniges Konstitutivum der Feier betrachtet. Doch führt er den „Weg Roms“ nicht einfach zu Ende, sondern erreicht einen qualitativen Neuansatz, indem die Einsetzungsworte nun ganz zur Verkündigung werden (daher laut und im Evangelienton rezitiert, so in der Deutschen Messe von 1526), zur „Anrede Christi an die gegenwärtig feiernde Gemeinde“ (460). Sie hören damit nicht auf, Konsekrationsworte zu sein, Worte über Brot und Wein, die dieses Brot und diesen Wein zur Gabe von Fleisch und Blut Christi für die Gemeinde machen. Dieser Gabecharakter sollte noch deutlicher hervortreten durch die weitere Funktion als Distributionsworte, also direkte Spendeformel in der Deutschen Messe. Dort sollten das Brot- und das Kelchwort jeweils der Austeilung der beiden Elemente direkt vorausgehen. Für die liturgische Praxis hat sich dieser Vorschlag nicht bewährt, doch der Intention nach blieb er in der lutherischen Messe bestehen (daher die Umkehrung der Reihenfolge von Einsetzungsworten und Vater Unser, noch heute in der Zweiten Form innerhalb der Grundform I des „Gottesdienstbuches“). Der Dank wird damit natürlich nicht ausgeschlossen, er ist im Gegenteil selbstverständlicher „Teil des Gottesdienstes, ja des christlichen Lebens überhaupt“, doch sind „Lob und Dank keine spezifischen Elemente der Abendmahlsfeier“ (465, meine Hervorhebung). Im Abendmahl „kann Sache der Kirche nichts anderes sein als der Empfang“ (464). Es ist das Verdienst reformatorischer Theologie, dies mit aller wünschenswerten Klarheit herausgearbeitet zu haben, und so besteht keinerlei Veranlassung, davon etwas aufzugeben.

Die These ist bewusst und gekonnt pointiert formuliert, und so mag es nicht verwundern, dass Widerspruch nicht ausblieb. Von systematisch-theologischer Seite wies U. Kübn darauf hin, dass hier „die Funktion der Kirche [...] eine komplexere und umfassendere [ist], als es die Rede vom ,reinen Empfangen“ zum Ausdruck bringt“ (264). Vielmehr seien das Reden und Handeln, das Beten und Danken der Menschen unauflöslich mit dem Handeln, mit der Gabe Gottes verwoben: Das „Ineinander“ von anabatischem und katabatischem Aspekt, „von geistgewirktem Dank der Gemeinde und je neuem Kommen Gottes“ (264) ist konstitutiv für das Herrenmahl. Ob die an dieser Stelle hergestellte Verbindung zur Rechtfertigungslehre und zur Rolle des Menschen dort besonders glücklich ist, mag dahin gestellt bleiben. Kühn hatte die von Wendebourg vorgelegte historische Rekonstruktion weit gehend unwidersprochen hingenommen und seine Kritik nur auf die konkreten liturgischen und theologischen Konsequenzen gerichtet.

Anders die beiden hauptsächlich Angegriffenen: H.-Ch. Schmidt-Lauber und F. Schulz. Nach einer knapperen Vorabpublikation des Erstgenannten erschien deren gemeinsame Replik 1999 im (katholischen) „Liturgischen Jahrbuch“. Da- 
rin werden sowohl der altkirchliche als auch der reformationshistorische Aspekt ausführlich besprochen. Überraschenderweise richtet sich der Widerspruch ganz überwiegend auf den zweiten. Zieht man die stark polemisch gefärbten Untertöne ab, so herrscht im Blick auf die Alte Kirche über weite Strecken Übereinstimmung: „Das Problem liegt nicht so sehr in der Erhebung der historischen Fakten als in deren Zusammenschau und Beurteilung." (97) Im Blick auf die Beurteilung mag eine gewisse Differenz allenfalls darin zu erkennen sein, dass Schmidt-Lauber (anders als Wendebourg) die Einsetzungsworte im Eucharistiegebet weniger schroff als Fremdkörper wahrnimmt (99). Beide Verfasser richten dagegen grundlegenden Widerspruch gegen die historischen Ausführungen zu Luther. Schmidt-Lauber diagnostiziert eine zu exklusive Orientierung am jungen Luther, an den bekannten Texten zur Gottesdienstreform der 20er Jahre, insbesondere ein Absolut-Setzen der „Deutschen Messe“ von 1526, die doch nur als eine Ausformungsvariante unter bestimmten Umständen gedacht gewesen sei und nicht gesetzlich isoliert werden dürfe (96).

Auch Schulz knüpft hier an und analysiert die Stellung und Deutung der Einsetzungsworte in der „Formula missae“ von 1523, in der "Deutschen Messe“ von 1526 und in weiteren einschlägigen Quellen des 16. Jh. (noch präziser: Schulz) Dabei kann er weder den ausschließlichen Verkündigungscharakter dieser Worte bestätigt finden noch ihre prinzipielle Isolation vom Gebetskontext. Es ist also „eine verengende Abgrenzung [...], wenn man das vielfach verschlungene Ineinander von Gottes Wort und Gebet, von Anrede und Antwort auseinander reißt, indem man das Verkündigen vom Beten, aber auch das Beten vom Verkündigen trennt" (113).

M. Meyer-Blanck versucht, den gordischen Knoten zu zerschlagen, indem er Wert und Gewicht historischer Argumentation insgesamt in ihre Schranken weist: „Evangelische Liturgiewissenschaft kann [...] ihre Normen nicht historisch, sondern nur von einer gegenwärtig verantworteten Aneignung des biblischen Evangeliums (von der Schriftauslegung) her begründen wollen“ (118). Indessen hatte weder Wendebourg noch sonst jemand behauptet, dass Liturgiewissenschaft aus der Historie Normen beziehe. Vor allem aber wird durch die erneute Aufrichtung eines Gegensatzes von Historie und biblischem Evangelium eine Form des Schriftprinzips repristiniert, die nach dem - für Vertreter aller Disziplinen unhintergehbaren - historischen Paradigma der Aufklärung und der Neuzeit kaum noch nachvollziehbar ist. Richtig und wichtig ist hingegen der Hinweis, dass sich die historische Argumentation nicht auf das 4 . und 16. Jh. beschränken darf und daher insbesondere nicht hinter die Liturgik Schleiermachers zurückgehen darf, der den Gottesdienst auf der Basis religiöser Erfahrung reflektierte. Daher kann „die soteriologische Vorordnung der Katabasis Gottes [...] inszenatorisch nur im Ineinander von Katabasis und Anabasis realisiert werden. Das Ineinander entspricht der Logik des religiösen Erlebens, die Überordnung der kritischen soteriologischen Reflexion“ (124, meine Hervorhebung). 
Der bislang letzte profilierte Beitrag zum Gespräch stammt aus der Feder des Neuendettelsauer Praktischen Theologen K. Raschzok. Eine detaillierte Bestandsaufnahme des bisherigen Diskussionsverlaufs verbindet er mit Beobachtungen über die Gründe für die harsche Kontroverse; er bietet insofern einen Metadiskurs. Auf der sachlichen Ebene betrachtet auch er „die Interpretation von Martin Luthers Messreform als entscheidende Weichenstellung“ (163). Er warnt vor einer Überfrachtung des historischen Quellenmaterials. Allenfalls könne man daraus „Kriterien für ein angemessenes Beten im Zusammenhang der Herrenmahlsfeier [ableiten], aber keinesfalls dessen Ausschluss aus den Konstitutiva der Feier [...] folgern“ (167). Im Blick auf den Diskussionsverlauf richtet er an Wendebourg den Vorwurf, sich als Kirchenhistorikerin „Übergriffe in das andere Fach“, nämlich die Liturgiewissenschaft, erlaubt zu haben. „Das Fach Kirchengeschichte erhebt damit implizit den Anspruch, zentrale Instanz in der Klärung dogmatischer und liturgischer Fragen zu sein und disqualifiziert darin die anderen Disziplinen der Theologie, insbesondere die Liturgiewissenschaft." (155) Umgekehrt werden aber auch der evangelischen Liturgiewissenschaft Vorwürfe nicht erspart: Sie „hat sich in den letzten Jahrzehnten möglicherweise zu selbstverständlich als Teil ökumenischer Liturgiewissenschaft verstanden und [...] das innerevangelische theologische Gespräch zu sehr vernachlässigt" (172).

In der Tat kann man sich des Eindrucks nicht erwehren, dass die streckenweise unerfreulichen Gesprächsverläufe nicht zuletzt mit Unzulänglichkeiten der theologischen Enzyklopädie bzw. der Fachaufteilung und Gesprächskultur im deutschsprachigen Protestantismus zu tun haben. Eine eigenständige Liturgiewissenschaft, die ihren Ort irgendwo in der Schnittmenge zwischen historischen, systematischen und praktischen Fragestellungen hat und die die damit verbundenen Spannungen (aber auch Reichtümer) in sich aushalten muss und darf, gibt es nicht (anders als in der katholischen Tradition). Das muss nicht unbedingt ein Nachteil sein, doch setzt es voraus, dass die jeweils nächstgelegenen Disziplinen sich ihrer besonderen Verantwortung gerade für diesen Teil der Theologie bewusst sind. Seine Bedeutung rückt besonders in den letzten Jahren wieder mehr ins Blickfeld der protestantischen Kirchen - das ist gut so, ja längst überfällig, doch darf akademische Theologie diese Tendenz nicht unter Verweis auf ihre jeweiligen Disziplinengrenzen ignorieren.

In diesem Sinne möge es dem Kirchenhistoriker abschließend gestattet sein, aus der Perspektive seines Faches, aber in der Verantwortung für das Ganze einige Beobachtungen inhaltlicher Art zu der skizzierten Diskussion vorzutragen.

1. Erklärte Absicht des Gesprächsbeitrags von Wendebourg war es, den „unsichtbaren Zaun“ zwischen Kirchengeschichte und Liturgiewissenschaft beiseite zu schieben (441). Unabhängig von der Frage, ob man diesen Versuch als gelungen ansieht, sollte man die Intention ernst nehmen, ja mit neuem Elan aufnehmen. Es ist keine Lösung, historische Argumente in theologischen Begründungszusammenhängen generell $\mathrm{zu}$ dispensieren, oder sich als Historiker auf 
den Posten des (angeblich) neutralen Beobachters zurückzuziehen. Fragen nach der Geschichte sind immer interessengeleitete Fragen: Es kommt nun darauf an, diese Interessen klar und explizit zu benennen und zum Teil der historischen Reflexion selbst zu machen. Dabei müssen „Übergriffe“ ins andere Fach jederzeit möglich sein; sie sind sogar erwünscht.

2. Es ist das Verdienst von Wendebourg, ein wichtiges Anliegen der Reformation, nämlich den Gabecharakter des Abendmahls wieder mit Nachdruck zur Geltung gebracht zu haben. Eine Antwort auf die Frage, welche liturgische Konsequenz aus dieser Einsicht zu ziehen ist, wird von ihr nicht (oder allenfalls umrisshaft und ad partem negativam) gegeben, und sie ist auch sonst in der Diskussion noch nicht hinreichend klar hervorgetreten.

3. Für einen Historiker ist es gewiss misslich, wenn er als entscheidenden Faktor der Entwicklung eine „verborgene Energie, von der der ganze [...] Prozess [...] zehrt“ (457), annehmen muss, etwas, das „schon immer da war“, so verborgen freilich, dass quellenmäßige Belege nicht oder nur in Andeutungen vorhanden sind. Alles Weitere erscheint dann als „Ausfaltung dessen, was von je her gegeben war“ (II, 419). Darin äußert sich ein latent teleologisches Geschichtsverständnis, das zumindest expliziert werden müsste: Es mag wohl sein, dass sich einem protestantischen Kirchenhistoriker der Verlauf so darstellt. Andere ex-post-Perspektiven würden andere Rekonstruktionen der Geschichte zulassen.

4. Die reformationshistorischen Thesen Wendebourgs sind bereits ausführlich diskutiert worden, der altkirchliche Teil der Argumentation bedarf noch weiterer Vertiefung. Insbesondere bleibt es problematisch, dass Ambrosius im Grunde der einzige klare Beleg für eine konsekratorische Wirkung der Einsetzungsworte in der Antike ist. Dennoch ist es auffällig, dass sich die Einfügung dieser Worte im 4. Jh. so gut wie überall in der Kirche durchgesetzt hat - offenbar in den meisten Fällen ohne das genannte Theologumenon. Bei näherem Hinsehen würde sich zeigen, dass es neben dem von Ambrosius vertretenen Deutungsversuch ein breites Spektrum von theologischen Motiven gibt, die keineswegs alle schon auf die Sichtweise des lateinischen Mittelalters (oder gar der Reformation) zulaufen. Es wäre dabei auf das geänderte Gottesdienstverständnis im 4. Jh. insgesamt zu verweisen - ein relativ komplizierter Prozess, bei dem auch frömmigkeitliche Entwicklungen und Einflüsse der umgebenden Religionen $\mathrm{zu}$ veranschlagen sind. Wollte man das Proprium dieses Gottesdienstverständnisses auf eine knappe Formel bringen, so könnte man von einer „historisierenden re-praesentatio“ sprechen. Ähnlich wie die Taufe nun mit dem Text Röm 6 liturgisch verbunden wird und dadurch Tod und Auferstehung vergegenwärtigt, wird auch im Abendmahl durch die verba testamenti „die Nacht, da er verraten ward, “ kultische Gegenwart (vgl. M. Wallraff, Von der Eucharistie zum Mysterium. Abendmahlsfrömmigkeit in der Spätantike: P. Gemeinhardt/ U. Kühneweg [Hg.], Patristica et Oecumenica, FS W. Bienert [MThSt 85], Marburg 2004, 89-104).

5. Solche Beobachtungen haben mit dem hier besprochenen Streit nicht un- 
mittelbar zu tun, sie sind aber dennoch weiterführend, weil beinahe alle liturgischen Reformbestrebungen erstens überhaupt altkirchliche Modelle verwenden und zweitens im Speziellen fast immer gerade das 4. Jh. als eine Art aetas aurea zugrunde legen - meist ohne sich selbst dieser Tatsache bewusst zu sein. Das gilt auch für die Kontrahenten des genannten Streites, und insbesondere die Verfechter des Eucharistiegebetes täten gut daran, sich zu erinnern, dass keiner von ihnen ein wirkliches „ad fontes“ vertritt, also eine Rückkehr zu Segensgebeten wie in der Didache oder in den Thomasakten (selbstverständlich ohne Einsetzungsworte). Diese archaische Eucharistie hat im 4. Jh. schon eine Fülle von zusätzlichen Sinnschichten um sich gelagert (und der Begriff selbst war übrigens schon wieder etwas zurückgetreten); es kann nur darum gehen, diese Sinnschichten differenziert wahrzunehmen und in ein reflektiertes Verhältnis zueinander zu setzen.

6. Ohnehin ist es wichtig, sich deutlich zu machen, dass die Kombination von theologischer Innovation und liturgischem Konservatismus gerade beim Abendmahl einen einzigartigen Prozess der Anreicherung von Motiven und Deutungen bewirkt hat. Die Liturgie als Liturgie ist ein vielschichtiges Zeichensystem erstaunlicher Komplexität - mit Zeichen verbaler und nonverbaler Art. Dass verschiedene Deutungen und Lesarten möglich und sinnvoll sind, macht ihren Reichtum aus. Der Satz vom ausgeschlossenen Widerspruch hat hier sein Recht, aber auch seine Grenzen. Auch literarische Brüche (etwa der Wechsel in der Anredeform) können historisch erklärbar und theologisch sinnhaft sein. Es mag sein, dass die Polarisierung und Differenzierung dieser Sinnebenen von einigem heuristischen Wert für den Theoriediskurs ist, doch ist dringend davor zu warnen, die dabei gewonnenen Erkenntnisse unmittelbar in liturgische Entwürfe zu übersetzen, die Liturgie also auf einen theologischen Entwurf hin gewissermaßen gleichzuschalten. Insofern sind Eucharistie und Herrenmahl gewiss keine Alternativen; es muss vielmehr darum gehen, die christliche Eucharistie als Herrenmahl zu feiern und zu reflektieren. 